\title{
Nutrición nitrogenada y tipo de híbrido y su influencia sobre atributos que determinan la aptitud bioenergética de sorgos
}

Torrecillas, M. G., Bertoia, L. M. y Jorge Rey, L.

\begin{abstract}
RESUMEN
El uso de fuentes de energía renovable está creciendo en el mundo y la búsqueda de biomasa alternativa para la producción de combustible, como el etanol, se ha intensificado. El objetivo de este trabajo fue estudiar el efecto de diferentes dosis de nitrógeno (N) sobre la eficiencia de uso y distintos atributos de la biomasa azucarada y lignocelulósica de cuatro morfotipos (Hib) de sorgo (Sorghum bicolor (L.) Moench). Durante dos campañas se evaluó el rendimiento de azúcar fermentable (RAF), rendimiento teórico de etanol a partir del jugo azucarado $\left(\mathrm{RE}_{\mathrm{azu}}\right.$ ), rendimiento de etanol celulósico $\left(R E_{c e}\right)$ y la eficiencia de uso de $N(E U N)$ para la producción de materia seca (RMS). Se detectó significancia del efecto año en la mayoría de las variables. La aplicación de $\mathrm{N}$ influenció sobre todas las variables estudiadas, excepto azúcares solubles (SST). La interacción NxHib no influyó significativamente sobre ningún atributo. Los morfotipos sileros se destacaron para RAF, debido a mayores valores de SST. La dosis de $50 \mathrm{~N}$ presentó el valor más alto de EUN. Si bien no existieron diferencias significativas entre morfotipos, la productividad de biomasa en morfotipos fotosensitivos fue determinante en la predicción del rendimiento de etanol, tanto a partir de azúcares como celulosa.
\end{abstract}

Palabras clave: Fertilización nitrogenada, sorgo dulce, aptitud bioenergética, etanol lignocelulósico.

Torrecillas, M. G., Bertoia, L. M. and Jorge Rey, L., 2018. Nitrogen nutrition and hybrid type and their influence on attributes that determine bioenergetic aptitude of sorghum. Agriscientia 35 (2): 1-9

\section{SUMMARY}

The use of renewable energy sources is growing worldwide and the search for alternative biomass for the production of fuel, such as ethanol, has intensified. 
The aim of this study was to evaluate the effect of different nitrogen $(\mathrm{N})$ rates on use efficiency and several attributes of the sugar and lignocellulosic biomass of four morphotypes (Hyb) of sorghum (Sorghum bicolor (L.) Moench). During two growing seasons, fermentable sugar yield (FSY), theoretical ethanol yield from fermentable sugar $\left(E Y_{\text {sug }}\right)$, cellulosic ethanol yield $\left(E Y_{\text {cel }}\right)$, and the nitrogen use efficiency (NUE) for dry matter yield (DMY) were evaluated. Significant year effects were observed for all measured traits. Nitrogen application strongly influenced all the traits measured, except soluble sugars (TSS). No significant $\mathrm{N}$ rate $\times$ morphotype interaction for any variable was noted. In both seasons sweet sorghum morphotypes were superior in FSY, due to higher values of soluble sugars (TSS). The $50 \mathrm{~N}$ rate presented the highest values in NUE. Although there were no significant differences between hybrids, the biomass yield of photosensitive types was decisive for ethanol yield prediction, both from sugars and cellulose.

Key words: Nitrogen fertilization, sweet sorghum, bioenergetic aptitude, lignocellulosic ethanol.

Torrecillas, M. G., Bertoia, L. M. y Jorge Rey, L. Facultad de Ciencias Agrarias, Universidad Nacional de Lomas de Zamora. Ruta 4, km 2 (1836), Llavallol. Correspondencia a: torrecillasm@yahoo.com.ar

\section{INTRODUCCIÓN}

El uso de fuentes de energía renovable está creciendo en el mundo y la búsqueda de biomasa alternativa para la producción de combustible, como el etanol, se ha intensificado.

La caña de azúcar y el sorgo dulce son cultivos tropicales que tienen la capacidad de almacenar azúcares fermentables en el tallo, que además como residuo sólido (bagazo) puede ser utilizado para la producción de energía eléctrica, biocombustible (etanol de segunda generación), alimento animal o fertilizante orgánico (Monti y Venturi, 2003; Ratnavathi et al., 2010).

El sorgo dulce es un cultivo con gran potencial bioenergético, debido a su rendimiento teórico de etanol, pero con menor energía invertida si se lo compara con maíz (Smith y Buxton, 1993; Hunter y Anderson, 1997). Además, el sorgo presenta alta productividad bajo condiciones desfavorables, debido a su tolerancia al estrés hídrico (Propheter, Staggenborg, Wu y Wang, 2010), suelos salinos, pobremente estructurados y temporariamente inundados (Houx, Roberts y Fritschi, 2013; Promkhambut, Polthanee, Akkasaeng y Younger, 2011); aun en esos ambientes, el sorgo tiene capacidad de producir lignocelulosa, azúcar y almidón (Rooney, Blumenthal, Bean y Mullet, 2007).

Existen dos tipos de sorgo que tienen alto potencial para la producción de bioenergía, los sorgos dulces y los fotosensitivos. En los primeros, la producción de etanol se obtiene a partir de jugo azucarado acumulado en los tallos, y del residuo celulósico de los tallos luego de la extracción de azúcar. Los híbridos forrajeros fotosensitivos están caracterizados por su gran altura de planta y los elevados rendimientos de materia verde. Debido a que requieren una duración del día de 12 h 20 ' para florecer, permanecen en estado vegetativo durante un período prolongado de tiempo (Tamang et al., 2011). Debido a que algunos híbridos tienen médula seca, pueden ser utilizados para la conversión a etanol celulósico a partir de valores adecuados de hemicelulosa y celulosa, aunque otros también tienen la capacidad de acumular jugo azucarado $y$, en estados avanzados de madurez, registrar altos valores de Brix (Rocatelli, Raper, Balkcom, Arriaga y Bransby, 2012; Smith, Allen y Barney, 2015).

Existen dos parámetros importantes que contribuyen a la potencialidad de la biomasa azucarada de un sorgo como fuente productora de bioetanol: su rendimiento en términos de volumen de jugo azucarado producido por hectárea, y la concentración y calidad de azúcares solubles de dicho jugo. No obstante, el potencial de híbridos sileros y fotosensitivos como fuente productora de azúcar no ha sido aún explotado del todo (Blumenthal, Rooney y Wang, 2007). 
Los principales componentes de la biomasa del sorgo son celulosa, hemicelulosa y lignina, y la demanda creciente de biomasa lignocelulósica para la producción de biocombustibles otorga valor al tejido vegetativo (Zhao et al., 2009). Ello conduce a un cambio de paradigma para optimizar la arquitectura de la planta en cultivos bioenergéticos ya que, por ejemplo, cobra importancia la altura de la planta y el diámetro del tallo para una determinada densidad de siembra.

La producción de etanol a partir de la fracción vegetativa es función de la cantidad de carbohidratos disponibles y de la convertibilidad de éstos, según los métodos usados para el pretratamiento y la hidrólisis (Dien et al., 2006).

Según Pedersen, Vogel y Funnell (2005) la reducción en el contenido de lignina (híbridos bmr) generalmente va acompañada por una concomitante reducción en la biomasa, y por consiguiente cualquier reducción de ésta debería estar compensada por un incremento en el rendimiento de azúcares totales fermentables.

Comparado con otros cultivos como el maíz, el rendimiento del sorgo no se ve gravemente afectado por la baja fertilidad de los suelos ni por problemas en sus condiciones físicas (Propheter et al., 2010; Houx et al., 2013). Tanto el sorgo dulce como el forrajero tienen menores requerimientos de $\mathrm{N}$ que el maíz, al mismo nivel de producción (Bennett y Anex, 2008; Han et al., 2012).

El requerimiento del cultivo en cuanto a la demanda de nutrientes es bajo al inicio del ciclo, pero la absorción de estos aumenta de manera significativa a partir del momento en que la planta tiene cinco o seis hojas verdaderas (esto sucede aproximadamente entre los 20 y 30 días posteriores a la emergencia) y se extiende hasta los 10 días previos a la floración. Por lo tanto, un buen aporte nutricional desde los primeros estados de desarrollo produce una cantidad suficiente de área foliar para interceptar mayor cantidad de radiación incidente, y asegurar así una alta eficiencia para transformarla en biomasa (Monk y Miller, 1984).

El nitrógenoes el nutrientecuya deficienciaesmás frecuente en las regiones sorgueras y su eficiencia de uso es trascendente para la sustentabilidad económica de los sistemas agrícolas. La mayoría de los nutrientes se encuentran disponibles a un $\mathrm{pH}$ entre 6 y 7 , aunque este no es el único factor que influye en su disponibilidad, particularmente en el caso del nitrógeno. Las condiciones ambientales como temperatura y humedad en el suelo pueden provocar importantes pérdidas posteriores a la aplicación de fertilizantes nitrogenados. Si bien existen algunos antecedentes de respuesta a
$\mathrm{N}$ en sorgo para planteos de producción de grano, es escasa la información acerca de los requerimientos, niveles de respuesta e impacto del $\mathrm{N}$ sobre azúcares y carbohidratos estructurales en sorgos dulces y fotosensitivos, cuando el objetivo es optimizar la producción de etanol.

El presente experimento fue conducido para estudiar el efecto de diferentes dosis de fertilización nitrogenada sobre distintos atributos de la biomasa azucarada y lignocelulósica, determinantes en la producción de etanol, sobre sorgos de morfología contrastante.

\section{MATERIALES Y MÉTODOS}

Los experimentos se llevaron a cabo en el Campo Experimental de la Facultad de Ciencias Agrarias de la Universidad Nacional de Lomas de Zamora (FCA-UNLZ) (34 49' 54,15" S; 58 43' 20,53" O), ubicado en el deslinde del partido de Cañuelas (provincia de Buenos Aires), durante las campañas 2013-2014 y 2014-2015.

Los ensayos fueron sembrados en ambas campañas en el período del 15 al 20 de noviembre. La preparación del terreno fue bajo labranza convencional, sobre un suelo con $3,2 \%$ de $\mathrm{MO}$; $38,1 \mathrm{ppm}$ de $\mathrm{P}$ extractable y $42 \mathrm{ppm}$ de $\mathrm{No}_{3}{ }_{3}^{-}$en 2013-2014 y 3,0 \%, 18,8 ppm y 33 ppm para 2014-2015, respectivamente, cuyos cultivos antecesores fueron maíz y sorgo silaje, en cada campaña, respectivamente. La siembra se efectuó con maquinaria experimental. Se realizó un control preventivo de malezas aplicando $4 \mathrm{I}$ ha $^{-1}$ de atrazina [6-cloro-N-etil-N'-(1-metil-etil)-1,3,5-triazina-2,4diamina] + 1,2 I ha- ${ }^{-1}$ de Dual Gold ${ }^{\circledR}$ (s-metolaclor) como tratamiento de preemergencia. Toda la semilla sembrada fue tratada con Concep $1{ }^{\Theta}$.

Los híbridos fueron seleccionados a partir de diferencias en la arquitectura de la planta y características morfofisiológicas. Los materiales fueron: ADV2800 ${ }^{\circledR}$ (fotosensitivo bmr), Green Feed ${ }^{\circledR}$ (fotosensitivo convencional), Sugargraze ${ }^{\circledR}$ (silero de baja proporción de grano) y TOB80SIL ${ }^{\circledR}$ (silero de alta proporción de grano).

Se evaluaron tres dosis de nitrógeno: 50, 100 y $150 \mathrm{~kg} / \mathrm{ha}$ de $\mathrm{N}$ aplicado al voleo y en banda entre surcos, a los 30 días de la emergencia, comparados a un testigo $0 \mathrm{~kg} / \mathrm{ha} \mathrm{N}$.

Se utilizó un diseño experimental de tres bloques completamente aleatorizados y la parcela experimental consistió en seis surcos de 5,2 m de longitud, separados 0,5 m entre surcos.

El momento de corte fue ajustado en cada híbrido, en fotosensitivos (ADV2800 y Green Feed) 
se realizó cuando las plantas alcanzaron 0,9 m de amarillamiento basal, y en los restantes materiales se efectuó cuando alcanzaron el estado de grano pastoso duro $\left(E_{8.2}\right)$ (Torrecillas, Cantamutto y Bertoia, 2011).

La cosecha y recolección de muestras se efectuó sobre los cuatro surcos centrales.

La metodología de cosecha consistió en cortar $1 \mathrm{~m}$ lineal y, previa separación y descarte de la fracción panoja en aquellos híbridos que la tuvieran, se registró su peso. Este peso constituyó el rendimiento de materia verde de la fracción vegetativa por parcela. Se tomaron muestras de estas fracciones de cada parcela, se colocaron a estufa de ventilación forzada con una temperatura de $60{ }^{\circ} \mathrm{C}$ hasta alcanzar peso constante. De acuerdo con el porcentaje de materia seca obtenido (MS), se calculó el rendimiento de materia seca por unidad de superficie (RMS).

Previo a cada cosecha se realizó la medición de sólidos solubles totales (SST) en el jugo sobre tres plantas representativas y en la sección central del tallo, utilizando un refractómetro portátil digital (ATAGO PAL-1, Atago USA). La variable SST se expresó en ${ }^{\circ}$ Brix.

Sobre las muestras obtenidas de tallo y hojas se realizaron las siguientes determinaciones de calidad:

- Contenido de hemicelulosa (\%) (Hcel).

- Contenido de celulosa (\%) (Cel).

Calculados en base a las expresiones:

Hcel $=$ FDN-FDA

Cel $=$ FDA-LDA

Las determinaciones de contenido de fibra detergente neutro (FDN), fibra detergente acido (FDA) y lignina detergente acido (LDA) se efectuaron mediante la técnica de bolsitas filtrables en incubador ANKOM ${ }^{220}$ (ANKOM technology Corp., Fairport, NY) (Vogel, Pedersen, Masterson y Toy, 1999).

Se calculó el rendimiento de azúcar fermentable (RAF) total, a partir de SST y de acuerdo a la expresión de Corn (2009), rendimiento teórico de etanol a partir del jugo azucarado $\left(\mathrm{RE}_{\mathrm{azu}}\right)$ a través de Bess Model 20083.1 (Liska et al., 2009) y rendimiento de etanol celulósico $\left(R_{c e l}\right)$, calculado a partir de valores de hemicelulosa y celulosa, de acuerdo a la expresión de Zhao, Steinberger, Shi, Han y Xie (2012).

Se evaluó la eficiencia agronómica del fertilizante aplicado (EUN), para la producción de materia seca total, de acuerdo a Fageria y Baligar (2005) como:

$$
E U N_{\text {MS }}=\left(M_{\mathrm{f}}-\mathrm{MS}_{0}\right) / \mathrm{N}_{\mathrm{f}}
$$

Donde: $\mathrm{MS}$, materia seca total $\left(\mathrm{kg} \mathrm{ha}^{-1}\right)$; $\mathrm{f}$, tratamiento fertilizado; 0 , tratamiento testigo; $N_{f}$, dosis de $\mathrm{N}$ del fertilizante.

Se analizaron ambos años separadamente, donde se efectuó un ANOVA doble entre dosis e híbridos y las comparaciones entre medias se realizó por medio de un test de diferencias mínimas significativas (DMS) con un nivel de significancia de $p<0,05$.

Para todos los análisis estadísticos se utilizó el software InfoStat (Di Rienzo et al., 2013).

\section{RESULTADOS Y DISCUSIÓN}

\section{Datos climáticos}

La Tabla 1 muestra la distribución de precipitaciones para ambas campañas. La campaña 2013-2014 registró menor acumulación y una distribución diferente respecto de 20142015. Desde noviembre a febrero, lapso de tiempo que incluye al período crítico del cultivo, el registro varió de 366 a $550 \mathrm{~mm}$, respectivamente. La segunda campaña se posicionó por encima del valor histórico (30 años), con distribución similar pero con una oferta hídrica en el mes de enero proporcionalmente superior a la campaña 2013-2014. Estas condiciones determinaron la ausencia de un período de déficit hídrico marcado, y posibilitó que los híbridos no desencadenaran latencia.

Tabla 1.Distribución de precipitaciones $(\mathrm{mm})$ en el período crítico del cultivo y registro histórico de 30 años, para las campañas 2013-2014 y 2014-2015.

\begin{tabular}{cccc}
\hline \multirow{2}{*}{ Mes } & \multicolumn{2}{c}{ Campañas } & Histórico \\
\cline { 2 - 4 } & $2013-2014$ & $2014-2015$ & $(1961-1990)$ \\
Nov & 140 & 212 & 91,3 \\
Dic & 66 & 90 & 77,8 \\
Ene & 67 & 140 & 68,4 \\
Feb & 93 & 108 & 87,0 \\
Total & 366 & 550 & \\
\hline
\end{tabular}

Promediando entre ambas campañas, y considerando los materiales que panojaron, el material más temprano (TOB80SIL) alcanzó el estado de mitad de floración $\left(E_{6}\right) 78$ días después de emergencia, mientras que Sugargraze lo hizo en 90 días. La altura de planta de los híbridos participantes varió de 4,08 (ADV2800) a 2,50 m (TOB80SIL). Aun tratándose ADV2800 de un híbrido de elevada altura y con el rasgo bmr 
incorporado, sólo se observó baja incidencia de vuelco, lo cual no comprometió la evaluación de las parcelas experimentales.

Debido a que el efecto año fue significativo para la mayoría de las variables, se analizaron los efectos de dosis de $\mathrm{N}$ e híbrido en cada campaña por separado.

\section{Campaña 2013-2014}

En la campaña 2013-2014, sólo se detectó significancia en la interacción $\mathrm{N} \times \mathrm{Hib}$ para la variable Cel. Asimismo, la dosis de $\mathrm{N}$ influenció significativamente sobre todas las variables (Tabla 2), excepto SST, que sólo registró efectos significativos de tipo de híbrido. Se destacó el comportamiento de ambos sileros (TOB80SIL y Sugargraze) para SST, ya que ambos se diferenciaron de los materiales fotosensitivos (ADV2800 y Green Feed), casi duplicando los valores de Brix y poniendo de manifiesto la mejor aptitud de aquellos cuando se prioriza la biomasa azucarada

Para RAF, las tres dosis de N (50, 100 y 150) fueron significativamente superiores al testigo (ON), aunque sin diferencias entre ellas (Tabla 2). Además, y a pesar de una buena performance en SST, si bien TOB80SIL registró un valor de RAF de 32 \% superior a Green Feed, ello no fue estadísticamente significativo. Los resultados aquí hallados son similares a los presentados por Holou y Stevens (2012) y Erickson, Woodward y Sollenberger (2012), para un nivel similar de Brix, aun cuando en ambos experimentos se efectuó la remoción previa de la panoja.

Al efectuar el promedio entre híbridos, para RMS, las dosis 150 y $100 \mathrm{~N}$ superaron significativamente a $50 \mathrm{~N}$ con una diferencia aproximada de $5 \mathrm{tMS}$, similar a la encontrada entre la dosis de $50 \mathrm{~N}$ y el testigo $0 \mathrm{~N}$. Aunque no existió significancia entre híbridos, se observó cierta tendencia a menores valores de Green Feeden cada una de las dosis de N. No pudo detectarse en la presente campaña alguna incidencia negativa del rasgo bmr, presente en ADV2800, sobre la performance de RMS.

Además, las tres dosis de $\mathrm{N}$ superaron al testigo para $\mathrm{RE}_{\mathrm{azu}}$ aunque sin diferencias entre ellas. No hubo significancia entre híbridos, pero tanto Sugargraze como TOB80SIL tuvieron buen comportamiento ya que combinaron buenos valores de rendimiento de biomasa con muy buena performance para SST. Estos resultados se contraponen parcialmente con los hallados por Tamang et al. (2011), quienes habían detectado buen comportamiento en híbridos fotosensitivos convencionales en condiciones ambientales diferentes a las del presente experimento.

$\mathrm{Si}$ bien existen diferentes opiniones acerca del momento de cosecha para maximizar el rendimiento de etanol, se encontró coincidencia con lo informado por Zhao et al. (2009), que indican que el momento óptimo podría ser entre 20 y 40 días postantesis, cuando el objetivo sea la producción de etanol a partir de azúcar.

Paralavariable Hcel se encontró un ordenamiento errático de las dosis de $\mathrm{N}$, con sólo tres puntos porcentuales en el rango de valores, donde $100 \mathrm{~N}$ $(29,18 \%)$ superó al resto. El híbrido ADV2800 tuvo un comportamiento significativamente superior frente a los híbridos restantes. Ello estaría ligado a que los menores valores de lignina determinados por el rasgo bmr determinan magnitudes inferiores sobre FDA (datos no mostrados).

Si bien Green Feed se destacó con mayores valores de Cel en $150(33,99 \%)$ y 50 N (32,33\%), se observó una situación particular con una alta

Tabla 2. Campaña 2013-2014. Rendimiento de azúcar fermentable (RAF), Rendimiento de MS (RMS), Rendimiento teórico de etanol a partir de azúcares $\left(R E_{\text {azu }}\right)$, Rendimiento de etanol celulósico $\left(R E_{\text {cel }}\right)$, Eficiencia de uso de $N(E U N)$ en respuesta diferentes dosis de $N$ y análisis de varianza (ANAVA).

\begin{tabular}{|c|c|c|c|c|c|c|}
\hline Dosis N & RAF (t ha-1) & RMS (t ha-1) & $\mathrm{RE}_{\mathrm{azu}}\left(\mathrm{Lha}^{-1}\right)$ & Hcel (\%) & $\mathrm{RE}_{\mathrm{cel}}\left(\mathrm{Lha}^{-1}\right)$ & $\begin{array}{c}\text { EUN } \\
\left(\mathrm{kg} \mathrm{MS} \mathrm{kg}^{-1} \mathrm{~N}\right)\end{array}$ \\
\hline 0 & $2,97 \mathrm{~b}$ & $10,95 \mathrm{c}$ & 1689 b & $27,75 a b$ & 3387 c & - \\
\hline 50 & $4,76 \mathrm{a}$ & $15,57 \mathrm{~b}$ & $2702 \mathrm{a}$ & 26,49 b & $4597 \mathrm{~b}$ & $96,17 a$ \\
\hline 100 & $5,31 \mathrm{a}$ & $19,18 \mathrm{a}$ & 3019 a & $29,18 \mathrm{a}$ & $5744 \mathrm{a}$ & $82,30 a$ \\
\hline 150 & $5,80 \mathrm{a}$ & $20,47 \mathrm{a}$ & 3296 a & $26,45 b$ & 5946 a & $63,48 b$ \\
\hline Dosis N & * & * & * & * & * & * \\
\hline Hib & ns & Ns & ns & ns & Ns & ns \\
\hline DxHib & ns & Ns & ns & $\mathrm{ns}$ & Ns & ns \\
\hline
\end{tabular}

Valores promediados entre híbridos. Medias en una columna seguidas de la misma letra no difieren significativamente a nivel de 0,05. D, dosis de N; Hib, tipo de híbrido. Los niveles de diferencias estadísticas significativas son indicados como: ns, no significativo; *, significativo a nivel $p<0,05$. 
performance en $0 \mathrm{~N}(35,47 \%)$, que posiblemente contribuyó en gran magnitud a la interacción $\mathrm{N} \times \mathrm{Hib}$

Por otro lado, considerando $\mathrm{RE}_{\text {cel }} \mathrm{y}$ promediando entre híbridos, las dosis de 100 y 150 N se diferenciaron netamente de la de $50 \mathrm{~N}$ y respectivamente todas del testigo $(0 \mathrm{~N})$, en este sentido, la de $50 \mathrm{~N}$ superó significativamente al testigo en un $27 \%$. Aunque no se vio reflejado claramente en los resultados del primer año, y en referencia al híbrido bmr (ADV2800), cabe destacar que cuando el objetivo es la obtención de etanol de segunda generación, el menor contenido de lignina en la MS determinará menor demanda de energía para el proceso de hidrólisis del residuo lignocelulósico (Cotton, Burow, Acosta Martínez y Moore-Kucera, 2013).

Los híbridos evaluados no registraron diferencias para EUN y las dosis de $50 \mathrm{~N}$ y 100 $\mathrm{N}$ se diferenciaron significativamente de la de 150 $\mathrm{N}, 50 \mathrm{~N}$ resultó numéricamente superior al resto $\left(96,2 \mathrm{~kg} \mathrm{MS} \mathrm{kg}^{-1} \mathrm{~N}\right)$. Además, la variable siguió una tendencia decreciente con el aumento de las dosis a partir de $50 \mathrm{~N}$, lo cual es coincidente con lo reportado por Maw, Houx III y Fritschi (2017), con una situación ambiental similar, respecto al régimen hídrico en el período crítico del cultivo.

\section{Campaña 2014-2015}

En la campaña 2014-2015 no se observó significancia en la interacción N×Hib, encontrándose efectos significativos de $\mathrm{N}$ en todas las variables, excepto SST, donde sólo se observó efectos de híbrido. Asimismo, se registraron efectos significativos de $\mathrm{N}$ e híbrido en $\mathrm{RAF}, \mathrm{RE}_{\mathrm{azu}}$ ' Hcel RE cel $^{\text {. }}$ (Tabla 3).

Tanto Sugargraze como TOB80SIL lograron los mayores de valores de RAF en las tres dosis de $\mathrm{N}$, oscilando los valores entre 7,45 tha $^{-1}$ para Sugargraze y $100 \mathrm{~N}$ y 4,51 tha $^{-1}$ para TOB80SIL y $150 \mathrm{~N}$. El menor valor registrado correspondió a ADV2800 y $0 \mathrm{~N}\left(2,03\right.$ tha $\left.^{-1}\right)$ (Tabla 3).

La variable SST tuvo un patrón de variación y magnitud similar que en la campaña 2013-2014, donde los híbridos sileros dulces se destacaron con respecto a los fotosensitivos, con valores que oscilaron entre 14,58 y $12,9^{\circ}$ Brix para TOB80SIL y Sugargraze, respectivamente; contra 8,73 y 7,48 ${ }^{\circ}$ Brix para Green Feed y ADV2800 respectivamente (Tabla 4). En concordancia con lo reportado por Erickson et al. (2012), quienes también evaluaron diferentes dosis de $\mathrm{N}$, en el presente experimento tampoco se detectó influencia de contenidos crecientes de N sobre SST.
Tabla 3. Campaña 2014-2015. Rendimiento de azúcar fermentable (RAF), Rendimiento teórico de etanol a partir de azúcares $\left(\mathrm{RE}_{\mathrm{azu}}\right)$, Contenido de hemicelulosa (Hcel), Rendimiento de etanol celulósico $\left(\mathrm{RE}_{\text {cel }}\right)$ sobre cuatro híbridos de sorgo, en respuesta a diferentes dosis de $\mathrm{N}$.

\begin{tabular}{ccccc}
\hline Dosis N & ADV2800 & Green Feed Sugargraze & TOB80SIL \\
\hline \multicolumn{5}{c}{ RAF $\left(\mathrm{t} \mathrm{ha}^{-1}\right)$} \\
$\mathbf{0}$ & $2,03 \mathrm{bA}$ & $3,14 \mathrm{aA}$ & $3,33 \mathrm{bA}$ & $3,97 \mathrm{bA}$ \\
$\mathbf{5 0}$ & $2,61 \mathrm{abB}$ & $4,34 \mathrm{aAB}$ & $6,35 \mathrm{abA}$ & $5,66 \mathrm{abA}$ \\
$\mathbf{1 0 0}$ & $4,32 \mathrm{aC}$ & $4,92 \mathrm{aBC}$ & $7,45 \mathrm{aA}$ & $6,97 \mathrm{aAB}$ \\
$\mathbf{1 5 0}$ & $3,41 \mathrm{abA}$ & $4,75 \mathrm{aA}$ & $4,54 \mathrm{abA}$ & $4,51 \mathrm{abA}$ \\
\multicolumn{5}{c}{ RE $_{\text {azu }}\left(\mathrm{L} \mathrm{ha}^{-1}\right)$} \\
$\mathbf{0}$ & $1155 \mathrm{bA}$ & $1781 \mathrm{bA}$ & $1889 \mathrm{bA}$ & $2257 \mathrm{bA}$ \\
$\mathbf{5 0}$ & $1484 \mathrm{abB}$ & $2465 \mathrm{aA}$ & $3605 \mathrm{abA}$ & $3213 \mathrm{abA}$ \\
$\mathbf{1 0 0}$ & $2456 \mathrm{aC}$ & $2795 \mathrm{aBC}$ & $4234 \mathrm{aA}$ & $3957 \mathrm{aAB}$ \\
$\mathbf{1 5 0}$ & $1940 \mathrm{abA}$ & $2700 \mathrm{aA}$ & $2577 \mathrm{abA}$ & $2559 \mathrm{abA}$ \\
\multicolumn{5}{c}{ Hcel $(\%)$} \\
$\mathbf{0}$ & $27,73 \mathrm{bAB}$ & $30,04 \mathrm{aA}$ & $26,29 \mathrm{aAB}$ & $25,64 \mathrm{aB}$ \\
$\mathbf{5 0}$ & $27,74 \mathrm{bA}$ & $28,90 \mathrm{aA}$ & $25,70 \mathrm{aA}$ & $28,16 \mathrm{aA}$ \\
$\mathbf{1 0 0}$ & $32,67 \mathrm{aA}$ & $31,15 \mathrm{aA}$ & $29,25 \mathrm{aA}$ & $28,56 \mathrm{aA}$ \\
$\mathbf{1 5 0}$ & $30,83 \mathrm{abA}$ & $29,29 \mathrm{aA}$ & $24,60 \mathrm{aB}$ & $26,69 \mathrm{aAB}$ \\
\multicolumn{5}{c}{$\mathbf{R E}_{\text {cel }}\left(\mathrm{L} \mathrm{ha}{ }^{-1}\right)$} \\
$\mathbf{0}$ & $3581 \mathrm{bA}$ & $5387 \mathrm{bA}$ & $4210 \mathrm{bA}$ & $3539 \mathrm{bA}$ \\
$\mathbf{5 0}$ & $7054 \mathrm{aA}$ & $8066 \mathrm{aA}$ & $7621 \mathrm{aA}$ & $7050 \mathrm{aA}$ \\
$\mathbf{1 0 0}$ & $7688 \mathrm{aA}$ & $8441 \mathrm{aA}$ & $8156 \mathrm{aA}$ & $7042 \mathrm{aA}$ \\
$\mathbf{1 5 0}$ & $8621 \mathrm{aA}$ & $8730 \mathrm{aA}$ & $4776 \mathrm{bB}$ & $4775 \mathrm{bB}$ \\
\hline
\end{tabular}

Medias en una columna, dentro de una sección, seguidas por la misma letra minúscula y medias en una fila, dentro de una sección, seguidas por la misma letra mayúscula no difieren significativamente a nivel $p<0,05$.

Tabla 4. Sólidos solubles totales (SST), valores de dos campañas, sobre cuatro híbridos de sorgo y promediados entre dosis de N.

\begin{tabular}{|c|c|c|c|c|}
\hline Campaña & ADV2800 & $\begin{array}{l}\text { Green } \\
\text { Feed }\end{array}$ & Sugargraze & TOB80SIL \\
\hline \multicolumn{5}{|c|}{${ }^{\circ}$ Brix } \\
\hline $2013 / 14$ & $7,45 b$ & $7,10 \mathrm{~b}$ & $13,95 \mathrm{a}$ & $14,26 \mathrm{a}$ \\
\hline $2014 / 15$ & $7,48 \mathrm{~b}$ & $8,73 \mathrm{~b}$ & $12,90 \mathrm{a}$ & $14,58 \mathrm{a}$ \\
\hline
\end{tabular}

Medias en una fila seguidas por la misma letra no difieren significativamente a nivel de 0,05.

La dosis de $100 \mathrm{~N}\left(26,04 \mathrm{t} \mathrm{MS}^{-1}\right)$ se ubicó al tope del ranking (promediando entre híbridos), aunque sin diferenciarse significativamente de $50(24,39 \mathrm{t}$ $\left.\mathrm{MS}^{-1}\right)$ y $150 \mathrm{~N}\left(22,13 \mathrm{t} \mathrm{MS}^{-1}\right)$ para RMS.

En este sentido, la presencia del rasgo bmr determina en general un comportamiento inferior para RMS, ya que la merma en el contenido de lignina implica, en la mayoría de los casos, una declinación del rendimiento de biomasa con respecto a híbridos convencionales (Oliver, Pedersen, Grant y Klopfenstein, 2005). Pese a ello, la presencia de dicha característica en ADV2800 no impactó sobre la performance de RMS, con 
respecto a los otros híbridos (convencionales), en ambas campañas.

Tanto N como Hib tuvieron significancia sobre $\mathrm{RE}_{\text {AzU }}$ Sugargraze $(4234,8)$ y TOB80SIL $(3957,5 \mathrm{~L}$ ha $^{-1}$ ) en $100 \mathrm{~N}$, tuvieron el mejor comportamiento, aunque sin diferenciarse significativamente ambos en las dosis de $50 \mathrm{~N}\left(3605,3\right.$ y 3213,9 $\left.\mathrm{L} \mathrm{ha}^{-1}\right)$.

La variable Hcel fue afectada por $\mathrm{N}$ e Hib, donde ambos fotosensitivos (ADV2800 y Green Feed) presentaron los mayores valores para $100 \mathrm{~N}$ con 32,67 y $31,15 \%$, respectivamente.

La dosis de $\mathrm{N}$ no condicionó en forma decisiva el comportamiento de Cel, donde incluso hubo un comportamiento errático de los valores y donde la variación no tuvo significancia (27,62-31,35 \%).

Al igual que lo informado por Tamang et al. (2011), quienes compararon sorgos dulces y fotosensitivos, valores superioresde Hcel y RMS determinaron en nuestro experimento la mejor performance de Green Feed (Híbrido fotosensitivo convencional) para $\mathrm{RE}_{\text {cel }}$ en 2014-2015.

Considerando EUN, $50 \mathrm{~N}$ fue significativamente superior al resto con valor numérico (208 kg MS kg-1 N) por encima de 2013-2014 (Tabla 5), promediado entre híbridos, registrándose un valor extremo de $253 \mathrm{~kg} \mathrm{MS} \mathrm{kg}^{-1} \mathrm{~N}$ para Sugargraze y 50 $\mathrm{N}$. Estos valores tienen similitud con los obtenidos por Maw et al. (2017) en sorgos dulces altos con una dosis de $56 \mathrm{~N}$ (239 kg MS kg-1 N) y con una oferta hídrica adecuada.

Por otro lado, el valor de 120,5 kg MS kg-1 N registrado para la dosis de $100 \mathrm{~N}$ y promediado entre híbridos, es plenamente coincidente a lo registrado por Grennell (2014), en híbridos fotosensitivos y sileros, en un estado de desarrollo

Tabla 5. Campaña 2014-2015. Rendimiento de MS (RMS), contenido de celulosa (Cel), Eficiencia de uso de N (EUN) en respuesta diferentes dosis de $\mathrm{N}$ y análisis de varianza (ANAVA).

\begin{tabular}{cccc}
\hline Dosis N & RMS $\left(\mathrm{t} \mathrm{ha}^{-1}\right)$ & Cel (\%) & EUN $\left(\mathrm{kg} \mathrm{MS} \mathrm{kg}^{-1} \mathrm{~N}\right)$ \\
\hline $\mathbf{0}$ & $13,99 \mathrm{~b}$ & $30,02 \mathrm{a}$ & - \\
$\mathbf{5 0}$ & $24,39 \mathrm{a}$ & $31,35 \mathrm{a}$ & $208,0 \mathrm{a}$ \\
$\mathbf{1 0 0}$ & $26,04 \mathrm{a}$ & $27,62 \mathrm{~b}$ & $120,51 \mathrm{~b}$ \\
$\mathbf{1 5 0}$ & $22,13 \mathrm{a}$ & $29,89 \mathrm{a}$ & $54,28 \mathrm{~b}$ \\
& & ANAVA & \\
Dosis N & $*$ & $*$ & $*$ \\
Hib & $\mathrm{ns}$ & $\mathrm{Ns}$ & $\mathrm{ns}$ \\
D $\times$ Hib & $\mathrm{ns}$ & $\mathrm{Ns}$ & $\mathrm{ns}$ \\
\hline
\end{tabular}

$\overline{\text { Valores promediados entre híbridos. Medias en una columna }}$ seguidas por la misma letra no difieren significativamente a nivel de 0,05. D, dosis de N; Hib, tipo de híbrido. Los niveles de diferencias estadísticas significativas son indicados como: ns, no significativo; *, significativo a nivel $p<0,05$. vegetativo similar (130 días de emergencia) y para una situación ambiental equivalente a nuestras condiciones experimentales, en cuanto a régimen hídrico durante el período crítico del cultivo.

Valores superiores de eficiencia de uso (200$370 \mathrm{~kg} \mathrm{MS} \mathrm{kg}^{-1} \mathrm{~N}$ ) reportados por Olson et al.(2013) para $100 \mathrm{~N}$, están vinculados a un período de crecimiento cercano a los 180 días de emergencia y con híbridos de mayor tasa de remobilización desde hojas basales.

En coincidencia con lo hallado por Maw, Houx III y Fritschi (2016), resultó destacable la proporcionalidad de respuesta a $\mathrm{N}$ observada para RMS, en ambas campañas, donde los registros pluviométricos fueron contrastantes. Además, pese a que en la campaña 2013-2014 se registró una productividad de biomasa alrededor de $23 \%$ menor que en la 2014-2015, no se observó un comportamiento diferencial de los híbridos.

La aplicación de fertilizante nitrogenado impactó favorablemente en la mayoría de los rasgos estudiados, excepto el contenido de azúcares solubles. La mayor contribución del rendimiento teórico de etanol lignocelulósico $\left(R E_{C E L}\right)$, con relación al derivado de azúcares $\left(R E_{A Z U}\right)$, sugiere que los productores deberían ajustar las dosis de $\mathrm{N}$ para incrementar el rendimiento de MS y que además resulte en un incremento de azúcares fermentables para una mayor eficiencia de conversión.

\section{BIBLIOGRAFÍA}

Bennett, A.S. y Anex, R. P. (2008). Farm-gate production costs of sweet sorghum as a bioethanol feedstock. Trans. ASABE 51, 603-613.

Blumenthal, J. B., Rooney, W. L. y Wang D. (2007). Yield and ethanol production in sorghum genotypes.Agron Abstract [CD-ROM]. ASA, Madison, Wisconsin.

Boudet, A. M. (1998). A new view of lignification. Trends of Plant Science, 3, 67-71. https://doi.org/10.1016/ S1360-1385(97)01176-X.

Corn, R. J. (2009). Heterosis and composition of sweet sorghum.PhD. Diss. Soil and Crop Sciences. Texas A\&M Univ., College Station. https://http://oaktrust. library.tamu.edu/bitstream/handle/1969.1/ETDTAMU-2009-12-7409/CORN-DISSERTATION.pdf.

Cotton, J., Burow, G., Acosta Martínez,V. y Moore-Kucera J. (2013). Biomass and cellulosic ethanol production of forage sorghum under limited water conditions. Bioenergy Research, 6, 711-718. doi: 10.1007/ s12155-012-9285-0. 
Dien, B. S., Jung, H. J. G., Vogel, K. P., Casler, M. D., Lamb, J. F. S., Iten, L., Mitchell, R. B. y Sarath, G. (2006). Chemical composition and response to diluteacid pretreatment and enzimatics accharification of alfalfa, reed canary grass, and switchgrass. Biomass \& Bioenergy, 30,880-891. https://doi.org/10.1016/j. biombioe.2006.02.004.

Di Rienzo, J.A., Casanoves, F., Balzarini, M. G., González, L., Tablada, M. y Robledo, C.W.(2013). InfoStat versión 2013. Grupo InfoStat, FCA, Universidad Nacional de Córdoba. Argentina.

Erickson, J. E., Woodward, K. R. y Sollenberger, L. E. (2012). Optimizing sweet sorghum production for biofuel in the southeastern USA through nitrogen fertilization and top removal. Bioenergy Research, 5, 86-94. doi: 10.1007/s12155-011-9129-3.

Fageria, N. K. y Baligar, V.C. (2005). Enhancing nitrogen use efficiencyin crop plants. Advances in agronomy, 88, 97-185.

Grenell, J. L. (2014). Yield and carbon exchange of sorghum grown as advanced biofuel feedstock on abandoned agricultural land in southeastern Ohio. Thesis. Ohio Univ. Athens.

Han, K. J., Putnam, W. D., Alison, M. W., Harrell, D. L., Viator, H. P. y McCormick, M. E. (2012). Agronomic considerations for sweet sorghum biofuel production in the south-central USA. Bioenerg \& Research, 5, 748-758. https://doi.org/10.1007/s12155-012-9185-3.

Holou, R. A. Y. y Stevens, G. (2012). Juice, sugar and bagasse response of sweet sorghum (Sorghum bicolor (L.) Moench cv. M81E) to N fertilization and soil type. GCB Bioenergy, 4, 302-310. doi: 10.1111/j.17571707.2011.01126.x.

Houx, J. H., Roberts, C. A. y Fritschi, F. B. (2013). Evaluation of sweet sorghum bagasse as an alternative livestock feed. Crop Science, 53, 1784-1790. doi:10.2135/ cropsci2012.03.0190.

Hunter, E. L. y Anderson, I. C. (1997). Sweet sorghum. Horticulture Reviews, 21, 73-104.

Liska, A. J., Yang, H. S., Bremer, V. R., Klopfenstein, T. J., Walters, D. T., Erickson, G. E. y Cassman, K. G. (2009). Improvements in life cycle energy efficiency and greenhouse gas emissions of corn-ethanol. Journal of Industrial Ecology, 13, 58-74. https://doi.org/10.1111/ j.1530-9290.2008.00105.x

Maw, M. J. W., Houx III, J. H. y Fritschi, F. B. (2016). Sweet sorghum ethanol yield component response to nitrogen fertilization. Industrial Crops and Products, 84, 43-49. doi: 10.2134/agronj2016.01.0044.

Maw, M. J. W., Houx III, J. H. y Fritschi, F. B. (2017). Nitrogen use efficiency and yield response of high biomass sorghum in the lower Midwest. Agronomy
Journal, $\quad 109, \quad 1-7 . \quad$ http://dx.doi.org/10.1016/j. indcrop.2016.01.038.

Monk, R. L. y Miller, F. R. (1984). Sorghum improvement for energy production. Biomass, 6, 145-153. https:// doi.org/10.1016/0144-4565(84)90017-9.

Monti, A. y Venturi, G. (2003). Comparison of the energy performance of fibre sorghum, sweet sorghum and wheat monocultures in northern Italy. European Journal of Agronomy, 19, 35-43. https://doi.org/10.1016/ S1161-0301(02)00017-5.

Oliver, A. L., Pedersen, J. F., Grant, R. J. and Klopfenstein, T. J. (2005). Comparative effects of the sorghum bmr6 and bmr-12 genes: I Forage sorghum yield and quality. Crop Science, 45, 2234-2239. doi:10.2135/ cropsci2004.0644.

Olson, S. N., Ritter, K., Medley, J., Wilson, T., Rooney, W. L. y Mullet, J. E. (2013). Energy sorghum hybrids: Functional dynamics of high nitrogen use efficiency. Biomass \& Bioenergy, 56, 307-316. http://dx.doi. org/10.1016/j.biombioe.2013.04.028.

Pedersen, J. F., Vogel, K. P. y Funnell, D. L. (2005). Impact of Reduced Lignin on Plant Fitness. Crop Science, 45, 812-819. doi:10.2135/cropsci2004.0155.

Promkhambut, A., Polthanee, A., Akkasaeng, C. y Younger, A. (2011). Growth, yield and aerenchyma formation of sweet and multipurpose sorghum (Sorghum bicolor L. Moench) as affected by flooding with different growth stages. Australian Journal of Crop Science, 5, 954965.

Propheter, J. L., Staggenborg, S. A., Wu, X. y Wang, D. (2010). Performance of annual and perennial biofuel crops: Yield during the first two years. Agronomy Journal, 102, 806-814. doi:10.2134/agronj2009.0301.

Ratnavathi, C. V., Suresh, K., Vijay Kumar, B. S., Pallavi, M., Komala, V. V. y Seetharama, N. (2010). Study on genotypic variation for ethanol production from sweet sorghum juice. Biomass \& Bioenergy, 34, 947-952. https://doi.org/10.1016/j.biombioe.2010.02.002.

Rocatelli, A. C., Raper, R. L., Balkcom, K. S., Arriaga, F. J. y Bransby, D. I. (2012). Biomass sorghum production and components under different irrigation/tillage systems for the southeastern US. Industrial Crops and Products, 36, 589-598. https://doi.org/10.1016/j. indcrop.2011.11.007.

Rooney, W. L., Blumenthal, J., Bean, B. y Mullet, J. E. (2007).Designing sorghum as a dedicated bioenergy feedstock. Biofuels Bioproducts and Biorefining, 1, 147-157. https://doi.org/10.1002/bbb.15.

Sanderson, M. A., Jones, R. M., Ward, J. y Wolfe, R. (1992). Silage sorghum performance trial at Stephenville. Forage Research in Texas. Rep. PR-5018. Texas Agric. Exp. Stn., Stephenville. 
Smith, G.A. yBuxton, D. R. (1993). Temperate zone sweet sorghum ethanol production potential. Bioresource Technology, 43, 71-75. https://doi.org/10.1016/09608524(93)90086-Q.

Smith, L. L., Allen, D. J. y Barney, J. N. (2015). Yield potential and stand establishment for 20 candidate bioenergy feedstocks. Biomass \& Bioenergy, 73, 145154. https://doi.org/10.1016/j.biombioe.2014.12.015.

Tamang, P. L., Bronson, K. F., Malapati, A., Schwartz, R., Johnson, J. y Moore-Kucera, J. (2011). Nitrogen requirements for ethanol production from sweet and photoperiod sensitive sorghums in the southern high plains. Agronomy Journal, 103, 431-440. doi: 10.2134/ agronj2010.0288.

Torrecillas, M. G., Cantamutto, M. A. y Bertoia, L. M. (2011). Head and stover contribution to digestible dry matter yield on grain and dual-purpose sorghum crop. Australian Journal of Crop Science, 5, 116-122.

Vanderlip, R. L. (1993). How a sorghum plant develops. S3. Department of Agronomy, Kansas State University.
Vogel, K., Pedersen, J., Masterson, S. y Toy, J. (1999). Evaluation of a filter bag system for NDF, and IVDMD forage analysis. CropScience, 39, 276-279.

Zhao, Y. L., Dolat, A., Steinberger, Y., Wang, X., Osman, A. y Xie, G. H. (2009). Biomass yield and changes in chemical composition of sweet sorghum cultivars grown for biofuel.Field Crops Research, 111, 55-64. doi: 10.1016/j.fcr.2008.10.006.

Zhao, Y. L., Steinberger, Y., Shi, M., Han, L. P. y Xie, G. H. (2012).Changes in stem composition and harvested produce of sweet sorghum during the period from maturity to a sequence of delayed harvest dates. Valores promediados entre híbridos.Medias en una columna seguidas por la misma letra no difieren significativamente a nivel de 0,05. D, dosis de $\mathrm{N}$; Hib, tipo de híbrido. Los niveles de diferencias estadísticas significativas son indicados como: ns, no significativo; *, significativo a nivel $\mathrm{p}<0,05$. 